\title{
Keep mental health in mind
}

\author{
A surge in mental-health problems related to the COVID-19 pandemic is on the horizon. The time to prepare and \\ prevent another health-crisis wave is now.
}

A s the COVID-19 pandemic continues to spread around the world, efforts have-rightly so-focused on addressing immediate medical needs: slowing down viral transmission chains to reduce the number of cases, optimize health-systems capacity and prevent fatalities. Now, 4 months into the pandemic, and with the realization that the end of the crisis is nowhere near, a different dimension of public health emerges and requires urgent attention. That is the toll of the pandemic on people's mental health and well-being.

Studies of past outbreaks provide some insight into the detrimental effects of similar crises on population-wide mental health. The 2003 SARS epidemic was associated with a $30 \%$ increase in suicides in people over the age of 65; almost a third of healthcare workers reported probable emotional distress; and survivors were found to be at risk for post-traumatic stress disorder and depression. Mitigation strategies such as quarantine, although necessary to contain viral spread, have a negative psychological impact, such as causing post-traumatic stress symptoms, emotional disturbance, depression and insomnia. Job loss and financial struggles during global economic downturn-which are already happening with the current pandemic-have been associated with a long-lasting decline in mental health. The scale, pervasiveness and complexity of the stressors associated with the ongoing pandemic are unprecedented in recent times.

There is no accurate template for what is yet to come, but odds are that a surge in the prevalence of mental-health problem is on the horizon. In fact, a study of over a thousand healthcare workers who cared for patients with COVID-19 in China showed that a considerable proportion of participants reported symptoms of depression (50\%), anxiety (45\%), insomnia $(34 \%)$ and distress $(72 \%)$. The droves of people exposed to the frontlines of the pandemic, such as essential workers, people with underlying health issues, and patients with COVID-19 and their caregivers, as well as people with pre-existing mental-health conditions, have been rendered particularly vulnerable to poor mental-health outcomes.
The pandemic undoubtedly has created almost universal psychological distress. A plan to address the problem and to prevent the long-term mental-health deterioration of segments of the population at most risk is paramount at this point in the COVID-19 response.

In March 2020, the World Health Organization released recommendations for safeguarding mental health, which are tailored to the individual person and are designed for immediate considerations. Similar guidelines have been issued by governmental agencies in the USA, the UK and other countries. However, much more needs to be done. A comprehensive assessment of the prevalence, severity and nature of COVID-19-related mental-health challenges, as well as the factors that contribute to risk and resilience, across the general public and especially in at-risk populations, will enable governments to plan for the inevitable wave of mental-health problems and dedicate the necessary resources for targeted interventions. Research initiatives tackling COVID19 and mental health have tended to be small in scale and localized; however, this landscape is changing, and larger studies are now underway. A nationwide survey in China reported that about a third of respondents experienced psychological distress during the COVID-19 pandemic. Similar studies in the UK have also revealed increased feelings of anxiety and depression and widespread concerns about the effect of social isolation on well-being, both in the general population and among people with pre-existing mental-health conditions. A study by the National Institute of Mental Health was recently launched in the USA, in which participants are asked about mental-health symptoms and COVID-19-related stress in the form of a questionnaire every 2 weeks for up to 6 months. Global action to map the landscape and longitudinal effects of COVID-19 on mental health across the various stages of the pandemic must be initiated immediately.

One approach to preempt an onslaught of mental-health problems on already overburdened healthcare systems could be to switch from individual-based approaches to population-wide screening aimed at identifying people at elevated risk. New innovations in digital health could be leveraged to assess mental-illness risk in large populations. For example, smartphone-based surveys, coupled with automated machine-learning analyses, have shown promise in identifying patients who are at risk for developing post-traumatic stress disorder. Digital phenotyping, which uses passively collected data to flag early signs of mental illness, is also gaining momentum. The development of both approaches, however, is still in its infancy, and rigorous testing of performance is needed before such initiatives can be rolled out at scale. A more immediate alternative would be to integrate psychiatric screening into primary care, using validated instruments such as the Patient Health Questionnaire depression scale (PHQ-9) and the Generalized Anxiety Disorder scale (GAD-7) to identify initial symptoms of depression and anxiety and to enable early, targeted intervention.

Researchers specializing in psychology, psychiatry, behavioral and social science, and digital health, as well as healthcare providers, policymakers and other stakeholders, must work together and toward innovative and practical technologies to address the mental-health needs under the current pandemic condition. Collecting and analyzing large-scale, high-quality data has to be prioritized now so that tailored and effective mental-health services can be implemented to best mitigate the long-term mental-health consequences of the pandemic later.

As the crisis caused by the COVID-19 pandemic shifts from acute to protracted, attention must be paid to the potentially devastating effects on population-wide mental health and emotional and social well-being. There will be no easy solution, but high-quality research, coupled with recent innovations in digital health, could enable health services to offer proactive and tailored mental-health care for those in need.

Published online: 4 May 2020 https://doi.org/10.1038/s41591-020-0914-4 